\title{
Fast and Robust Clinical Triple-Region Image Segmentation Using One Level Set Function
}

\author{
Shuo Li ${ }^{1,2}$, Thomas Fevens ${ }^{2}$, Adam Krzyżak ${ }^{2}$, Chao $\mathrm{Jin}^{2}$, and Song $\mathrm{Li}^{3}$ \\ ${ }^{1}$ GE Healthcare, London, Ontario, Canada \\ shuo.li@ge.com \\ ${ }^{2}$ Department of Computer Science and Software Engineering, \\ Concordia University, Montréal, Québec, Canada \\ \{fevens, krzyzak, chao_jin\}@cs.concordia.ca \\ ${ }^{3}$ School of Stomatology, Anhui Medical University, Hefei, Anhui, P.R. China \\ xlisong@sohu.com
}

\begin{abstract}
This paper proposes a novel method for clinical triple-region image segmentation using a single level set function. Triple-region image segmentation finds wide application in the computer aided X-ray, $\mathrm{CT}, \mathrm{MRI}$ and ultrasound image analysis and diagnosis. Usually multiple level set functions are used consecutively or simultaneously to segment triple-region medical images. These approaches are either time consuming or suffer from the convergence problems. With the new proposed triple-regions level set energy modelling, the triple-region segmentation is handled within the two region level set framework where only one single level set function needed. Since only a single level set function is used, the segmentation is much faster and more robust than using multiple level set functions. Adapted to the clinical setting, individual principal component analysis and a support vector machine classifier based clinical acceleration scheme are used to accelerate the segmentation. The clinical acceleration scheme takes the strengths of both machine learning and the level set method while limiting their weaknesses to achieve automatic and fast clinical segmentation. Both synthesized and practical images are used to test the proposed method. These results show that the proposed method is able to successfully segment the triple-region using a single level set function. Also this segmentation is very robust to the placement of initial contour. While still quickly converging to the final image, with the clinical acceleration scheme, our proposed method can be used during pre-processing for automatic computer aided diagnosis and surgery.
\end{abstract}

\section{Introduction}

Due to the complexity of medical image segmentation and the requirements of the clinical environment, in terms of speed and accuracy, clinical image segmentation is one of most challenging topics in the computer aided medical image analysis and diagnosis.

The triple-region segmentation problem deals with the segmentation of images that naturally consist of three distinct regions of interest. Triple-region 
segmentation finds wide application in medical imaging due to the fact that most X-rays, CT, MRI and ultrasound images can be modeled as a triple-region segmentation problem. This paper reports on our work towards a robust and efficient clinical triple-region image segmentation method using one of the latest image processing techniques: the level set method.

Level set methods have been shown to be one of the most versatile techniques for medical image segmentation due to its ability to capture the topology of shapes in medical imagery. Although some work have been reported on medical image segmentation using level set 12 , there are many challenges remain in the application of level set for medical image segmentation, especially clinical image segmentation, even for triple-region images.

\section{Challenges}

\subsection{Coupled Versus Hierarchical}

The main problem of the level set representation lies in the fact that a level set function is restricted to the separation of two regions. As soon as more than two regions are considered, the level set idea loses much of its attractiveness. Only a few papers focus on level set based segmentation for more than two regions, which can be divided into the following two categories: coupled level set and hierarchical level set.

Coupled Level Set. Coupled level set employs multiple level set function simultaneously for multi-region segmentation. In [3, Zhao et al. assign one level set function to each region. The number of level set function is equal to the number of regions in the image. A very similar framework for classification was proposed by Samson in 4. Both techniques, however, assume an initially fixed number of regions. This assumption is omitted in [5] where the number of regions is estimated in a preliminary stage by means of a Gaussian mixture estimate of the image histogram. This way, the number of mixture coefficients determines the number of regions. A considerably different approach is proposed by Vese and Chan in [6]. By their method, the level set functions are used in such a way that $N$ regions are represented by only $\log _{2} N$ level set functions. Unfortunately, this will result in empty regions if $\log _{2} N$ is a float rather than a integer. These empty regions have undefined statistics, though the statistics still appear in the evolution equations. To segment a triple-region image using coupled level set method, three coupled level set functions are usually used. However in [7, Li et al. proposed a competitive level set which uses two coupled level set functions to segment triple regions. In general, the coupled level set suffers from the slow converge and local minimal problem.

Hierarchical Level Set. Hierarchical level set segmentation 89] employs an hierarchical approach to extend two region segmentation method to multiple region segmentation. The image is first segmented into two regions by one level set function. Then based on the variance analysis of each region, the program 
decides to further segment one or both regions. The procedure is done recursively until the whole image is properly segmented. Although comparing to coupled level set, hierarchical level set has advantage as easy implementation and fast segmentation. However for triple-region segmentation, it requires using two level set segmentation consecutively. And therefore the processing time is at least doubled.

As described above, the prominence of current level set based segmentation is lost as soon as more than two regions come into play. The purpose of this paper is to address this problem for a particular image segmentation problem: tripleregion medical image segmentation using level set framework while preserving its advantages.

\subsection{Clinical Segmentation Using Level Set}

Moreover as indicated in [10, although efficient, level sets are not suitable for clinical segmentation. This is not only because it is usually a time consuming method, when coupled with complicated medical structure, even using only three coupled level set functions can be very time consuming, but also because of the following factors: level set is sensitive to the placement of initial contour. Therefore the running time of level set method heavily relies on the position and size of the initial curves and the complexity of objects, coupled level set functions do not converge for some initial curves and for some cases, different initial contours may give different segmented results. A good level set based clinical image segmentation method should be able to overcome all of these problems.

\section{Proposed Framework}

To overcome the above problems, we propose a novel idea of using single level set function to segment the three regions. The proposed level set functional formulates triple-region segmentation as two dual-region segmentation. Since only one level set function is used, it is much faster and robust, comparing to existing level set method.

To adapt the processing in clinical setting, we combine the strength of pattern classifier and level set to achieve a robust and fast clinical segmentation for three regions medical images. A pattern classifier and individual principal component analysis are used to accelerate the pathological level set segmentation. The evolution of the level set function achieves a fast and robust triple-region segmentation since the initial contour provided by the SVM and PCA is very close to the final contour.

\subsection{Level Set Modelling}

For a clear description, the three regions are named as bright region (BR), grey region (GR), and dark region (DR) as shown in the Fig. 1. According the Mumford and Shah modelling, the correct segmentation can be obtained by minimizing energy function given as: 


$$
\begin{gathered}
E(\Phi)=\lambda_{1} \iint_{\Omega_{B R}} \frac{\left(u-c_{B R}\right)^{2}}{\sigma_{B R}^{2}} d x d y+\lambda_{2} \iint_{\Omega_{G R}} \frac{\left(u-c_{G R}\right)^{2}}{\sigma_{G R}^{2}} d x d y \\
+\lambda_{3} \iint_{\Omega_{D R}} \frac{\left(u-c_{D R}\right)^{2}}{\sigma_{D R}^{2}} d x d y
\end{gathered}
$$

where $c_{i}$ and $\sigma_{i}$ are mean intensity value and variance of the region $\Omega_{i}$ and $\lambda_{i}$ is a constant.

To formulate the three regions segmentation problem into two region segmentation framework, a new energy function is proposed as shown in Eq. 2. By the functional proposed, the triple-region segmentation is naturally formulated simultaneously as solving two dual region segmentation problems: grey region versus dark region and grey region versus bright region.

$$
E(\Phi)=\lambda_{1} \iint_{\Omega_{-}} \operatorname{Min}\left(\frac{\left(u-c_{B R}\right)^{2}}{\sigma_{B R}^{2}}, \frac{\left(u-c_{D R}\right)^{2}}{\sigma_{D R}^{2}}\right) d x d y+\lambda_{2} \iint_{\Omega_{+}} \frac{\left(u-c_{G R}\right)^{2}}{\sigma_{G R}^{2}} d x d y
$$

where the function $\operatorname{Min}(x, y)$ returns the smaller value of $x$ and $y$.

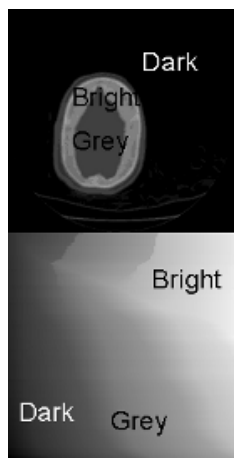

Fig. 1. Triple-region modelling

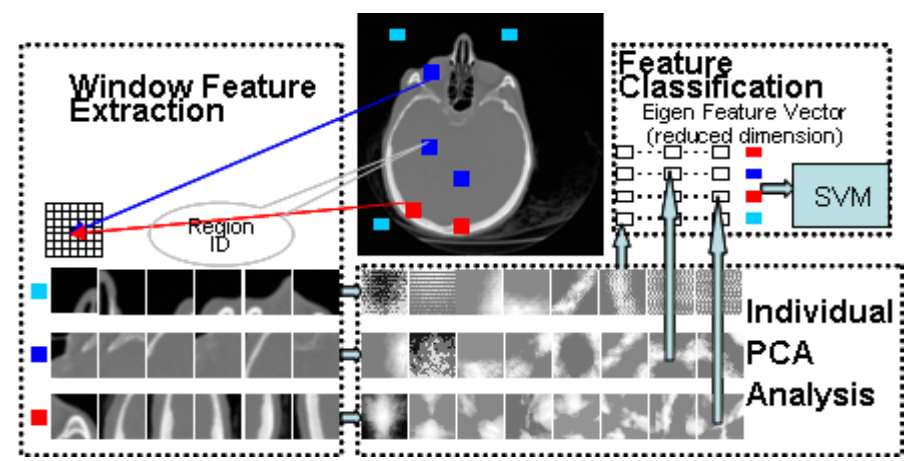

Fig. 2. Feature extraction diagram

To achieve fast and robust segmentation, a hybrid level set functional that combines minimal variance (Eq. ??), the optimal edge integrator [10] and the geodesic active contour model [10] is used:

$$
E=E(\Phi)-\gamma_{1} E_{L A P}+\gamma_{2} E_{G A C},
$$

where $\gamma_{i}$ are constants, the geodesic active contour $\left(E_{G A C}\right)$ and edge functional $\left(E_{L A P}\right)$ are defined in Eq. 4

$$
\begin{gathered}
E_{G A C}(C)=\iint g(C) d x d y \\
E_{L A P}(C)=\int_{C}<\nabla, \mathbf{n}>d s+\iint_{\Omega_{C}} K_{u}|\nabla u| d x d y .
\end{gathered}
$$

Here $K_{u}$ is the mean curvature of the level set function, $\mathbf{n}$ is the unit vector normal to the curve and $d s$ is the arc length of curve $C$. 
Function $g(x, y)$ is an inverse edge indicator function, which is defined as $g(x, y)=\alpha^{2} /\left(\alpha^{2}+|\nabla u|^{2}\right)$, where $\alpha$ is a constant and $\nabla$ is the gradient operator. The level set function $\Phi$ is derived from the functional in Eq. 3 as shown:

$$
\begin{gathered}
\frac{\partial \Phi}{\partial t}=\delta_{\varepsilon}(\Phi)\left[\gamma_{2} \operatorname{div}\left(g \frac{\nabla \Phi}{|\nabla \Phi|}\right)-\lambda_{2} \frac{\left(u-c_{G R}\right)^{2}}{\sigma_{G R}^{2}} H(\Phi)-\gamma_{1} u_{\xi \xi}\right. \\
\left.+\lambda_{1} \operatorname{Min}\left(\frac{\left(u-c_{B R}\right)^{2}}{\sigma_{B R}^{2}}, \frac{\left(u-c_{D R}\right)^{2}}{\sigma_{D R}^{2}}\right)(1-H(\Phi))\right],
\end{gathered}
$$

where $H(\cdot)$ is the Heaviside function, $\operatorname{div}(\cdot)$ is the divergence operator, and $u_{\xi \xi}=\Delta u-K_{u}|\nabla u|$.

\subsection{Clinical Acceleration}

Designed for the dental clinical setting, the segmentation has a clinical acceleration scheme, which divides the segmentation into two stages: a training stage and a clinical segmentation stage. During the training stage, first, manually chosen representative images, which consist of three regions, are segmented by the level set segmentation method proposed. Then the results are used to train a support vector machine (SVM) after PCA. During the clinical segmentation stage, images are first classified by the trained SVM after PCA, which provides initial contours. The evolution of the level set function will give the final segmentation result. Instead of using global PCA, we use the individual PCA approach described in [11, since it is able to reduce more dimensions than global PCA. The feature extraction process is described in Fig. 2.

\section{Experimental Results}

Both a synthesized image and real medical images (CT and X-ray) are used to test the proposed method. The results are shown and analyzed below.

\subsection{Segmentation}

First the proposed level set segmentation method is tested using a synthesized triple-region image as shown in Figs. 3, and 4. The original image consists of three Gaussian distributed regions. As shown in the Fig. 3, the proposed level set method successfully segments the grey region from the dark region and bright region with one level set function only. Compared to using three level set functions,

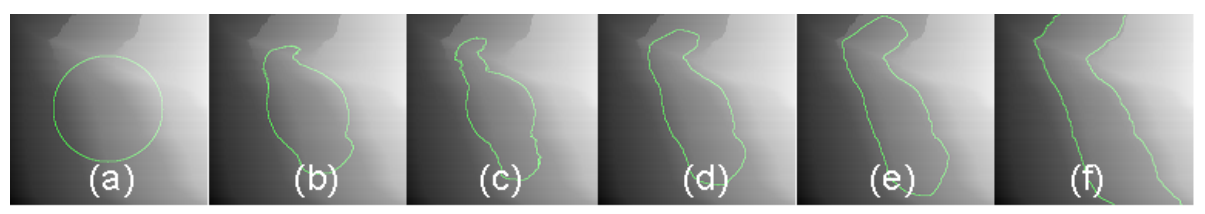

Fig. 3. Segmentation results on synthesized triple-region image. (a) Iteration 0. (b) Iteration 10. (c) Iteration 20. (d) Iteration 40. (e) Iteration 60. (f) Iteration 80. 

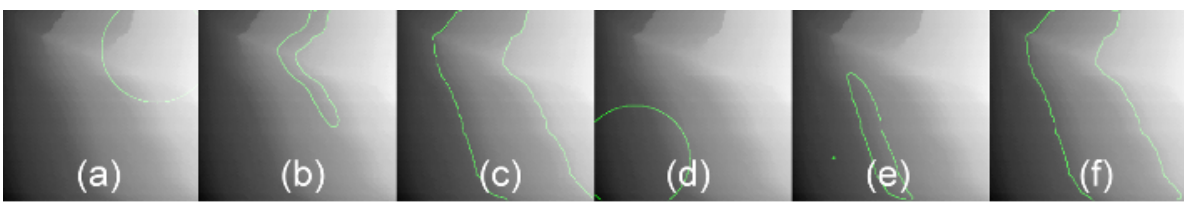

Fig. 4. Segmentation results on synthesized triple-region image with different initial contours. (a) Iteration 0. (b) Iteration 60. (c) Iteration 100. (d) Iteration 0. (e) Iteration 60. (f) Iteration 100.

one level set function is much faster and more robust. Moreover the segmentation method is robust to position of the initial contour. Regardless of where the initial contour is placed, where you put the initial contour, the level set still able to converge to the final correct segmentation as shown in Figs. 3 and 4 .

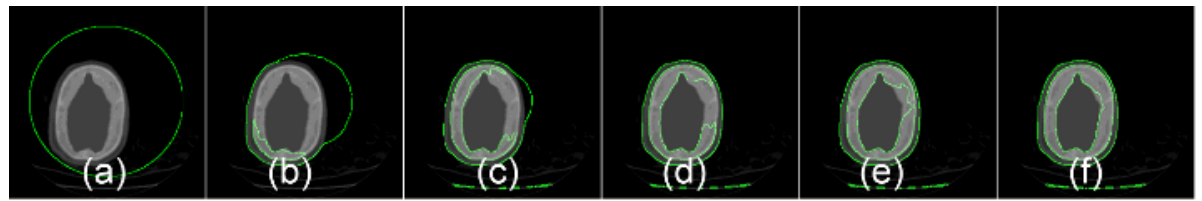

Fig. 5. Segmentation results on CT image with contour of middle region. (a) Iteration 0. (a) Iteration 10. (c) Iteration 20. (d) Iteration 40. (e) Iteration 60. (f) Iteration 80.

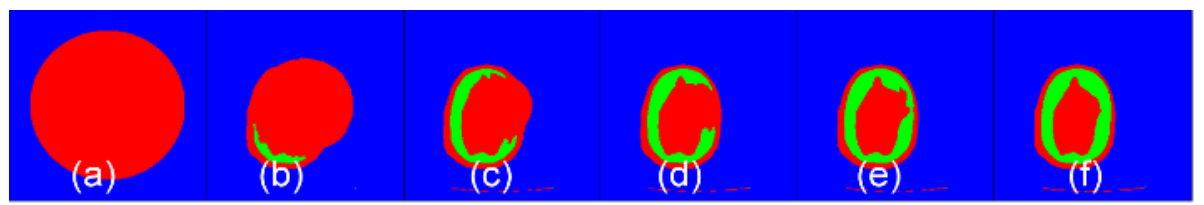

Fig. 6. Segmentation results on CT image. (a) Iteration 0. (a) Iteration 10. (c) Iteration 20. (d) Iteration 40. (e) Iteration 60. (f) Iteration 80.

Figs. 5 to 7 show segmentation results on CT head scan. Fig. 5 shows the evolution of zero contour and Fig. 6] shows the region segmentation in correspondent to zero curve evolution. Fig. 7 shows the contour and region evolution on another slice of scan. Figs. 8(a-c) show the segmentation results on an astrophysics
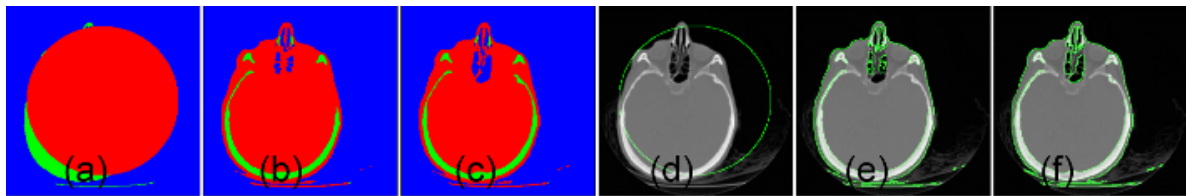

Fig. 7. (a) Iteration 0. (b) Iteration 30. (c) Iteration 50. (d) Iteration 0. (e) Iteration 30. (f) Iteration 50. 


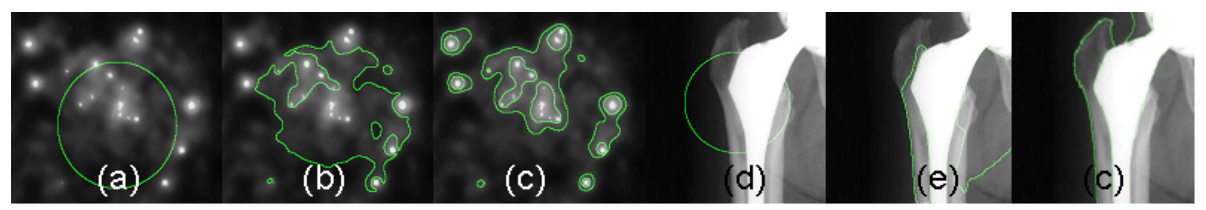

Fig. 8. (a) Iteration 0. (b) Iteration 50. (c) Iteration 90. (d) Iteration 0. (e) Iteration 50. (f) Iteration 90.

X-ray. As we can see, even though the image is very noisy and boundaries are also very unclear, the segmentation can still be achieved. Figs. 8(d-f) show the segmentation results on a bone X-ray.

\subsection{Clinical Acceleration}

Fig. 9 shows the clinical acceleration results. Since the PCA and SVM are able to provide initial boundaries closes to the final boundaries, it takes only few iterations to achieve the final segmentation as shown in the comparison of Figs. 3, 4, 6) and 9. Since that individual PCA is able to reduce the dimension to $10 \%$ of the original dimension, which greatly speed up the SVM training and classification. This makes the proposed segmentation method easily being used in the real time application.
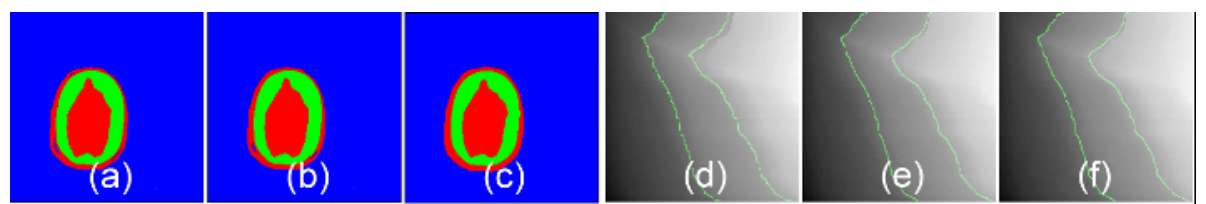

Fig. 9. Segmentation results on using clinical acceleration. (a) Initial contour provided by SVM . (b) Iteration 5. (c) Iteration 15. (d) Initial contour provided by SVM. (e) Iteration 5. (f) Iteration 15.

\section{Conclusion}

To facilitate the application of the level set method in clinical triple-region image segmentation, a single level set function based segmentation method is proposed. The method is based on the boundary characteristics of the triple regions. By the new proposed energy modelling, the triple-region segmentation is put into the framework of dual regions segmentation. Using the functional proposed, the triple-region segmentation is naturally formulated as two dual region segmentation problems. The clinical image segmentation acceleration used takes the strengths of both machine learning and the variational level set method while limiting their weaknesses to achieve automatic and fast clinical segmentation. Both a synthesized image and real images are used to test the proposed method. 
Experimental results show that the proposed method is able to provide a fast and robust clinical triple-region image segmentation using only one single level set function. The proposed method can be used as pre-processing step for automatic computer aided diagnosis. The proposed method can be also easily extended to multiple region segmentation using a hierarchical scheme.

\section{References}

1. J. ha An, Y. Chen, F. Huang, D. C. Wilson, and E. A. Geiser, "A variational pde based level set method for a simultaneous segmentation and non-rigid registration.," in MICCAI, pp. 286-293, 2005.

2. P. Yan and A. A. Kassim, "Mra image segmentation with capillary active contour.," in MICCAI, pp. 51-58, 2005.

3. H.-K. Zhao, T. F. Chan, B. Merriman, and S. Osher, "A variational level set approach to multiphase motion," Journal of Computational Physics, vol. 127, no. 1, pp. 179-195, 1996.

4. C. Samson, L. Blanc-Fraud, G. Aubert, and J. Zerubia, "A level set model for image classification," International Journal of Computer Vision, vol. 40, no. 3, pp. 187-197, 2000.

5. N. Paragios and R. Deriche, "Coupled geodesic active regions for image segmentation: A level set approach," in ECCV'00: Proceedings of the 6th European Conference on Computer Vision-Part II, (London, UK), pp. 224-240, Springer-Verlag, 2000 .

6. L. Vese and T. Chan, "A multiphase level set framework for image segmentation using the Mumford and Shah model," International Journal of Computer Vision, vol. 50, no. 3, pp. 271-293, 2002.

7. S. Li, T. Fevens, A. Krzyżak, C. Jin, and S. Li, "Semi-automatic decay detection on dental x-rays using variational level set," in Medical Image Computing and Computer-Assisted Intervention (MICCAI), vol. 3749 of Lecture Notes in Computer Science, (Palm Springs, California, USA), pp. 670-678, Springer, 2005.

8. M. Jeon, M. Alexander, W. Pedrycz, and N. Pizzi, "Unsupervised hierarchical image segmentation with level set and additive operator splitting," Pattern Recognition Letters, vol. 26, pp. 1461-1469, July 2005.

9. A. Tsai, J. Yezzi, A., and A. Willsky, "Curve evolution implementation of the Mumford-Shah functional for image segmentation, denoising, interpolation, and magnification," IEEE Trans. on Image Processing, vol. 10, pp. 1169 - 1186, 2001.

10. S. Li, T. Fevens, and A. Krzyżak, "Image segmentation adapted for clinical settings by combining pattern classification and level sets.," in Medical Image Computing and Computer-Assisted Intervention (MICCAI), vol. 3216 of Lecture Notes in Computer Science, (St-Malo, France), pp. 160-167, Springer, 2004.

11. X. Liu, T. Chen, and B. V. K. V. Kumar, "Face authentication for multiple subjects using eigenflow.," Pattern Recognition, vol. 36, no. 2, pp. 313-328, 2003. 